\title{
Double-Rate Tomlinson-Harashima Precoding for Multi-Valued
} Data Transmission

\author{
Yosuke IIJIMA $^{\dagger \text { a) }}$ and Yasushi YUMINAKA ${ }^{\dagger \dagger}$, Members
}

SUMMARY The growing demand for high-speed data communication has continued to meet the need for ever-increasing $\mathrm{I} / \mathrm{O}$ bandwidth in recent VLSI systems. However, signal integrity issues, such as intersymbol interference (ISI) and reflections, make the channel band-limited at high-speed data rates. We propose high-speed data transmission techniques for VLSI systems using Tomlinson-Harashima precoding (THP). Because THP can eliminate ISI by inverting the characteristics of channels with limited peak and average power at the transmitter, it is suitable for implementing advanced low-voltage and high-speed VLSI systems. This paper presents a novel double-rate THP equalization technique especially intended for multi-valued data transmission to further improve THP performance. Simulation and measurement results show that the proposed THP equalization with a double sampling rate can enhance the data transition time and, therefore, improve the eye opening.

key words: multi-valued data transmission, equalization, TomlinsonHarashima precoding, high-speed interface, serial link

\section{Introduction}

The demand for higher bandwidth chip-to-chip and boardto-board interconnection technologies has been increasing significantly because data centers require aggregate $\mathrm{I} / \mathrm{O}$ bandwidth to handle huge amounts of data. However, in such high-speed serial links, channel distortions arising from intersymbol interference (ISI), the skin effect, dielectric loss, and reflections severely limit I/O bandwidth relative to the device performances. This is because interconnections such as micro-strip lines (MSLs) on printed circuit boards (PCBs) act as low-pass filters. As shown in Fig. 1, the low-pass effect of interconnections introduces ISI, which causes bit errors due to the difficulty of $\mathbf{0}$ or $\mathbf{1}$ detection at the receiver. Such ISI can be countered by applying inverse channel responses using signal processing (e.g., preemphasis, continuous time linear equalizers (CTLEs) and decision feedback equalizers (DFEs)) [1], [2].

In our previous work [3]-[5], we presented a highspeed data transmission technique for VLSI systems using Tomlinson-Harashima precoding (THP) as a transmitter equalizer. Because THP can eliminate ISI by inverting the characteristics of channels with limited peak and average power at the transmitter, it is suitable for implementing advanced low-voltage and high-speed VLSI system communi-

\footnotetext{
Manuscript received October 18, 2016.

Manuscript revised February 20, 2017.

Manuscript publicized May 19, 2017.

The author is with the National Instutute of Technology, Oyama College, Oyama, 323-0806 Japan.

${ }^{\dagger \dagger}$ The author is with the Graduate School of Science and Technology, Gunma University, Kiryu-shi, 376-8515 Japan.

a)E-mail: yiijima@ oyama-ct.ac.jp

DOI: 10.1587/transinf.2016LOP0014
}

cation. We also demonstrated THP-based multi-valued data communication on a MSL to show its feasibility.

In this paper, we present novel double-rate equalization using THP, hereinafter referred to as a doublerate THP. Double-rate THP employs double-rate sampling points, which can enhance the edge of the data transition, resulting in improved signal integrity. We experimentally demonstrate that double-rate THP could mitigate the ISI of multi-valued data transmissions more clearly compared to conventional THP. In addition, an adaptive coefficient calibration technique for THP is considered to alleviate the variation in the channel characteristics.

The remainder of this paper is organized as follows. Section 2 explains high-speed data transmission using THP. In Sect. 3, we present double-rate equalization using THP, which is the new proposal in this paper. In Sect. 4, the experimental results are presented. In Sect. 5, we discuss the impact of double-rate THP and the adaptive coefficient calibration method. Section 6 concludes the paper.

\section{Tomlinson-Harashima Precoding}

\subsection{PAM-4 High-Speed Data Transmission Using} Tomlinson-Harashima Precoding

Figure 2 shows an overview of the THP-based data trans-

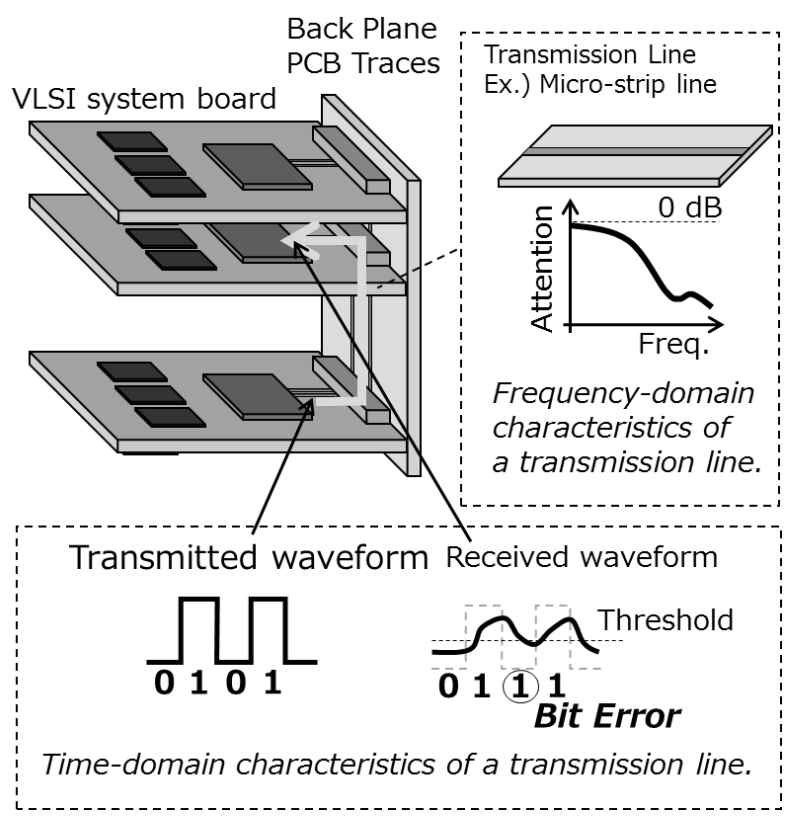

Fig. 1 High-speed data transmission of VLSI systems and the ISI effect. 


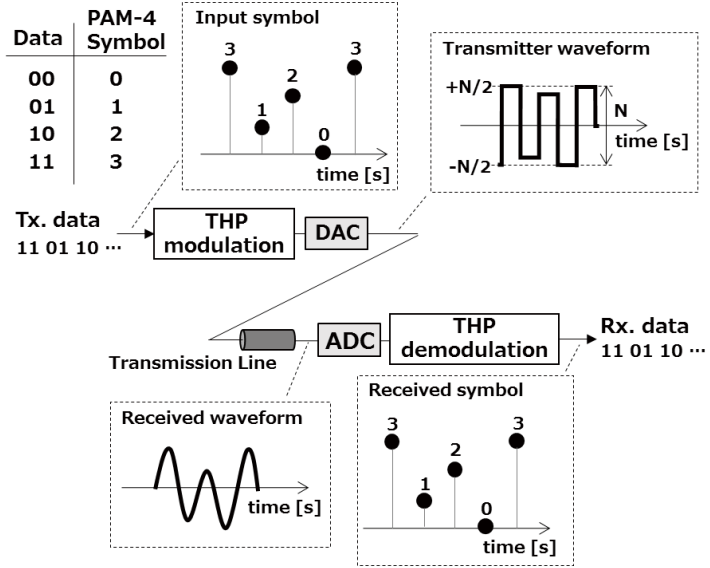

(a) THP-based data transmission

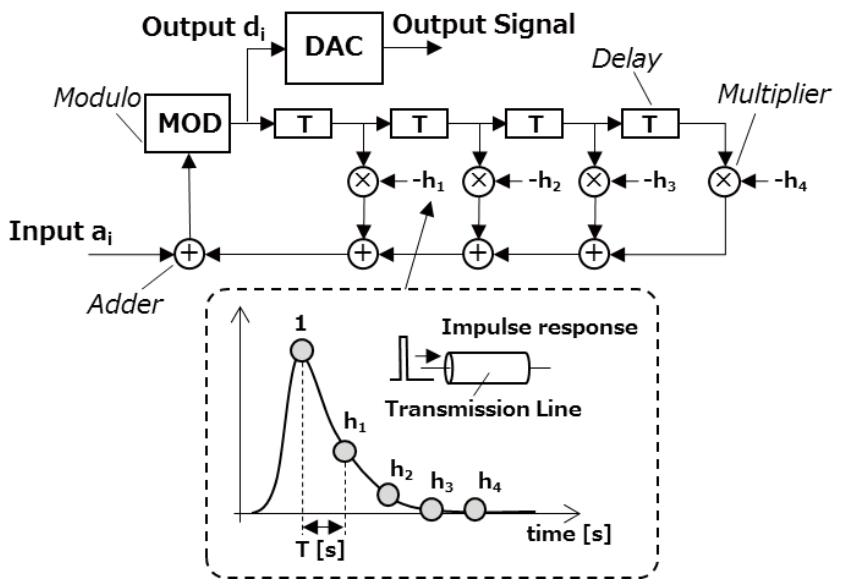

(b) Block diagram of THP

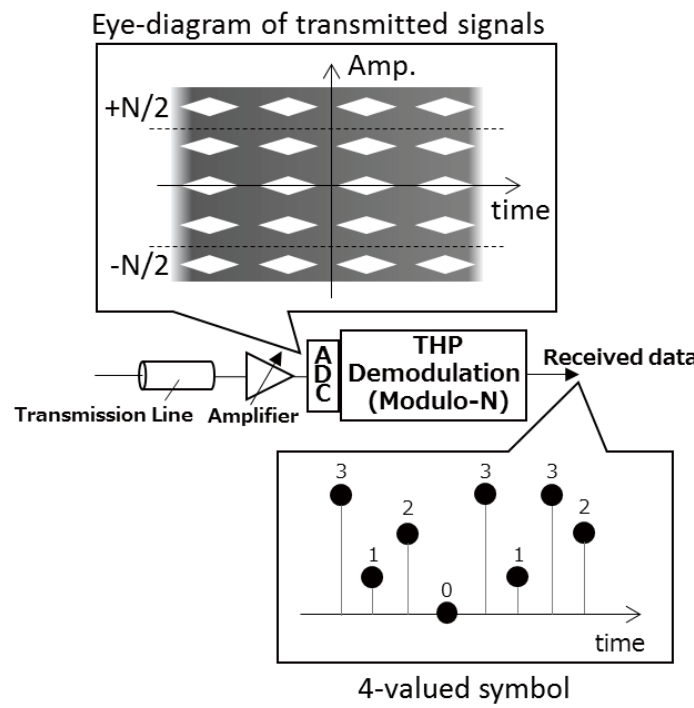

(c) Demodulation of THP at a receiver

Fig. 2 Overview of a THP-based data transmission system (PAM-4 data transmission). mission system. In this system, we employ a multiplevalued coding technique that handles greater than two-level signaling amplitudes to represent data. For example, a 4 -valued (PAM-4) coding represents two-bit information $(\mathbf{0 0 / 0 1 / 1 0 / 1 1 )}$ as one symbol $(\mathbf{0} / \mathbf{1} / \mathbf{2} / \mathbf{3})$ using four-level signaling. The 4 -valued signaling can reduce the symbol rate by 2 compared to binary signaling using four-level amplitudes, therefore lowering the channel bandwidth requirements. THP is a digital equalizer that can remove an ISI via digital signal processing at a transmitter [6], [7]. The THP can be realized via a digital filter that uses a modulo- $N$ computing unit (Fig. 2(b)), where $T$ indicates the symbol rate of the input data. Because THP can limit the output peak power for the transmission signal between $-N / 2$ and $+N / 2$ using modulo- $N$ operation, it is suitable for low-voltage and highspeed data transmission. The THP modulated transmitter waveforms are generated via a digital-to-analog converter (DAC). At the receiver, after sampling data via an analogto-digital converter (ADC) and modulo- $N$ reduction, we can recover the input data.

The data symbols $a_{i}(i=1,2, \ldots)$ are modulated at the transmitter. The THP output signals $d_{i}(i=1,2, \ldots)$ are

$$
d_{i}=\left(a_{i}-\sum_{j=1}^{n} h_{j} d_{i-j}\right) \text { modulo } N
$$

and, therefore, the magnitude of $d_{i}$ is limited between $-N / 2$ and $+N / 2$. As shown in Fig. 2(b), the THP coefficients $h_{1}, \ldots, h_{n}$ can be calculated from the the sampled values of the impulse response of the channel at the transmission data rate as

$$
H(z)=1+h_{1} z^{-1}+h_{2} z^{-2}+h_{3} z^{-3}+\cdots+h_{n} z^{-n} .
$$

The number of taps $n$ of THP depends on the channel characteristics of $H(z)$, especially the post-cursor length. It is necessary to obtain the impulse response of the transmission line in advance to design the THP circuit. By folding the THP output by modulo- $N$ operations, the transmitter symbol levels are increased in some cases. However, the folded data symbol is recovered by modulo- $N$ reduction at the receiver (Fig. 2(c)). In the following simulations and experiments, we use an eye diagram in terms of eye height and eye width to evaluate the signal integrity.

\subsection{Evaluation of Data Transmission Using THP on MSL}

Figure 3 shows the measured S21-parameter frequency characteristics of $1 \mathrm{~m}$ and $2 \mathrm{~m}$ MSLs that were fabricated on an FR-4 substrate for evaluation. We simulated the received waveforms via a numerical analysis using measurement S-parameter results of the frequency characteristics of the MSLs.

Figures 4 and 5 are the simulation results of eye diagrams of PAM-4 2 Gsps (symbols per second) data transmission without and with THP on $1 \mathrm{~m}$ and $2 \mathrm{~m}$ MSLs, respectively. As shown in Figs. 4 and 5, the THP can reduce the ISI at the timing of the symbol decision in the receiver, 


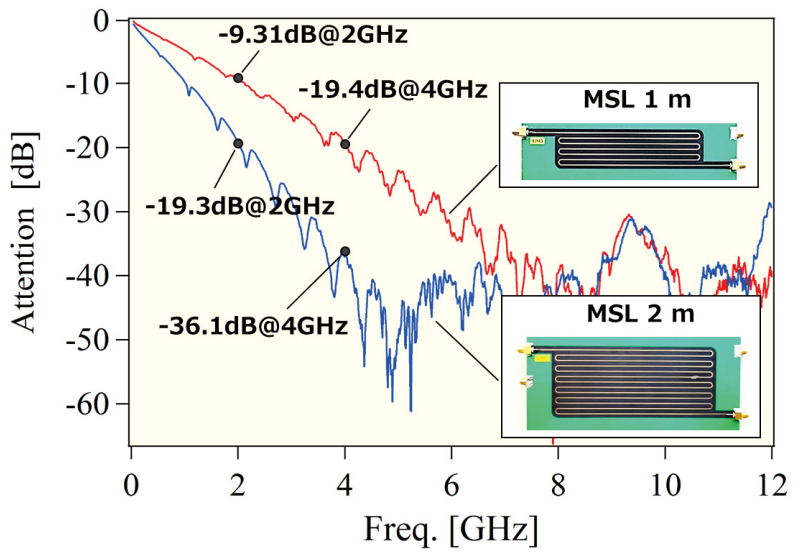

Fig. 3 Measured frequency characteristics of the fabricated $1 \mathrm{~m}$ and $2 \mathrm{~m}$ MSLs.

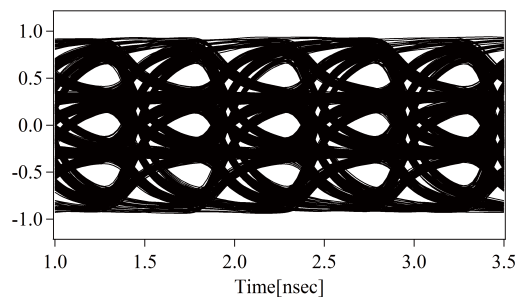

(a) Without THP

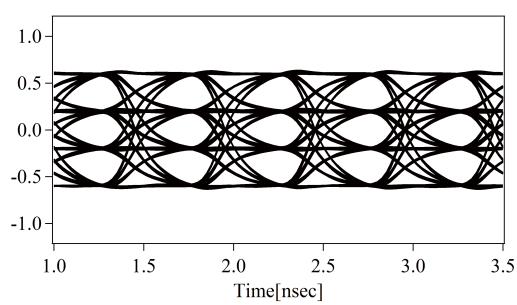

(b) With THP

Fig. 4 Simulation results of an eye diagram at a receiver for multi-valued data transmission (a) without and (b) with THP (PAM-4 signaling at 2 Gsps on a $1 \mathrm{~m} \mathrm{MSL}$ ).

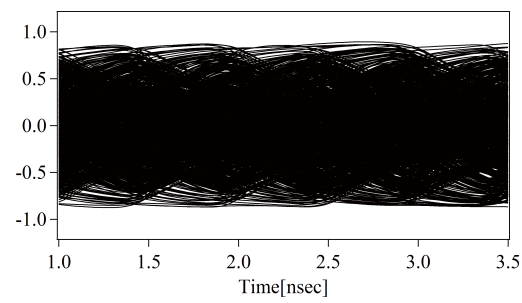

(a) Without THP

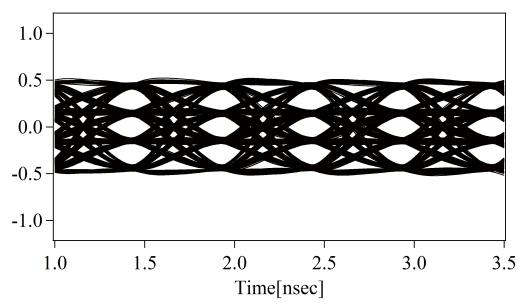

(b) With THP

Fig.5 Simulation results of an eye diagram at a receiver for multi-valued data transmission (a) without and (b) with THP (PAM-4 signaling at 2 Gsps on a $2 \mathrm{~m} \mathrm{MSL}$ ). widely opening the eye height.

As the data rate increases, the rise and fall times and the jitter become larger, resulting in increased distortion and eye closure. Even though THP can remove the ISI, it is difficult to expand the eye width for multi-valued data transmission. In the following section, to improve eye width, a novel double-rate equalization technique using THP for multi-valued data transmission is proposed.

\section{Double-Rate Equalization with THP}

The bock diagram of the double-rate THP is shown in Fig. 6 (a). The double-rate THP circuit configuration has an inverse filter operated at the double frequency of the symbol

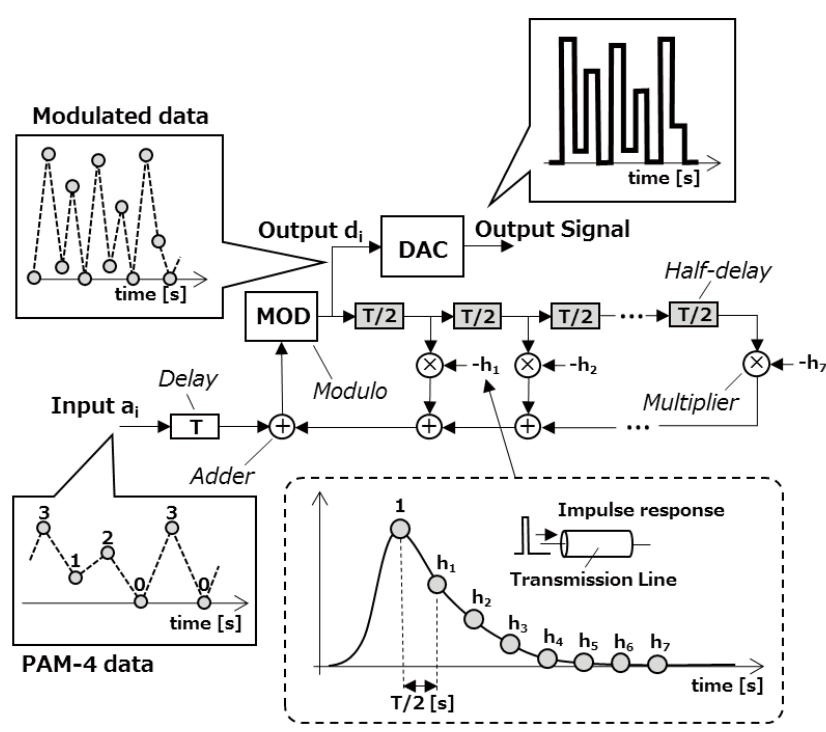

(a) Block diagram of a double-rate THP

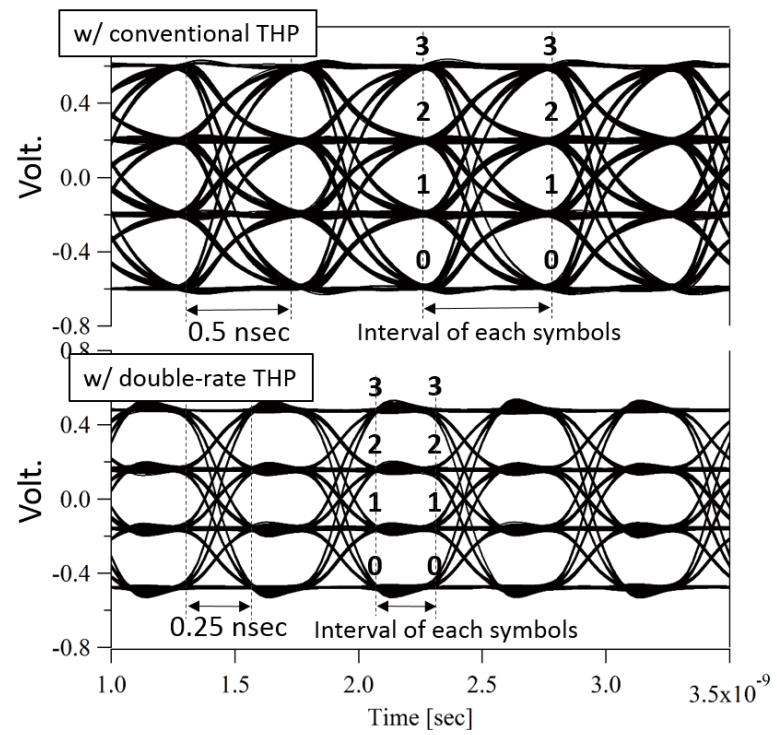

(b) Simulation results of received waveforms at 2 Gsps on a $1 \mathrm{~m}$ MSL (PAM-4) using conventional THP and double-rate THP

Fig. 6 Overview of double-rate THP. 
rate $(2 \times 1 / T \mathrm{~Hz})$ compared to conventional THP. As shown in Fig. 6(a), the coefficients $h_{1}, h_{2}, \ldots, h_{n}$ are determined from the impulse responses, which are sampled at half of the symbol cycle $(T / 2 \mathrm{sec})$. Therefore, the number of taps is increased compared to conventional THP (Fig. 2(b)). In double-rate THP, transmitter data is held for $T$ sec by a delay element. Then, the inverse filter generates a modulated signal at the double symbol rate $(2 \times 1 / T \mathrm{sps})$.

Figure $6(\mathrm{~b})$ shows the simulation results of PAM-4 data transmission using double-rate THP at 2 Gsps on a 1 $\mathrm{m}$ MSL. Because the transition time between each symbol level is reduced to approximately half that of the conventional THP (e.g., ref.[8]), the eye width can be greatly expanded. As shown in Fig. 6(b), the PAM-4 data transition pattern has many combinations compared to that of binary; therefore, the eyes have gentle slopes that decrease the eye width. By contrast, double-rate THP can sharpen the transition edge of the data using double sampling points that make it possible to improve the PAM-4 eye width. As a result, double-rate THP can reduce ISI more clearly compared to conventional THP.

\section{Experimental Results}

As a proof of concept, we apply THP to high-speed data transmission on MSLs. Figure 7 shows the experimental system setup for the double-rate THP. AWG70001A (Tektronix) and MSO73304DX (Tektronix) are used as the arbitrary waveform generator and sampling oscilloscope, respectively. As a 6-tap 8-bit THP transmitter, the arbitrary waveform generator produces a THP-modulated random pulse pattern, which is applied to the MSL. To calculate the THP-modulated signal using the THP coefficients, the measured S-parameter of the MSL was used. Then, a sampling oscilloscope observes the transmitted waveform and draws the eye diagram.

Figure 8 shows the measured eye diagrams of 2 Gsps PAM-4 data transmission without and with THP on a $1 \mathrm{~m}$

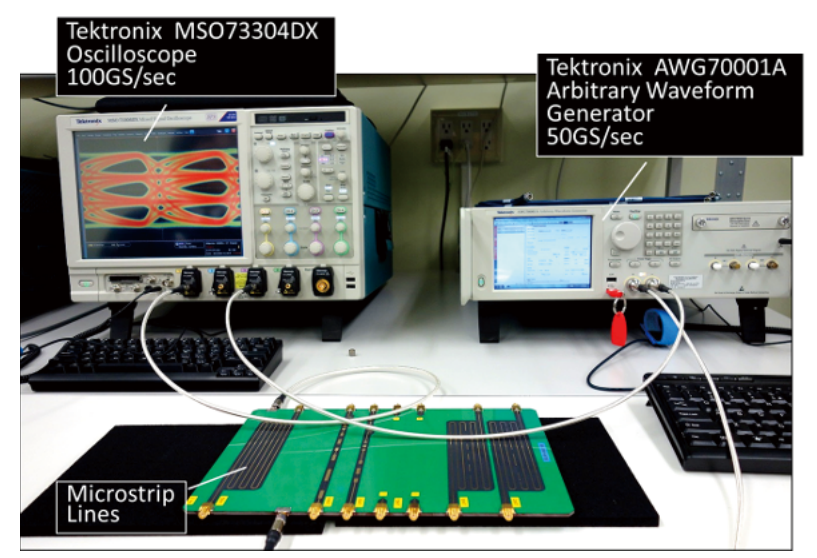

Fig. 7 Measurement system setup. An arbitrary waveform generator AWG70001A is used to transmit THP-modulated data for transmission by the MSL. Output waveforms are observed using a sampling oscilloscope MSO73304DX.
MSL. Figures 8(b) and 8(c) show eye diagrams of the conventional and double-rate THPs, respectively. The eye width of the conventional THP (Fig. 8(b)) is 0.46 UI. Here UI corresponds to a unit interval, which corresponds to the symbol rate of the data. Using double-rate THP, we can reduce

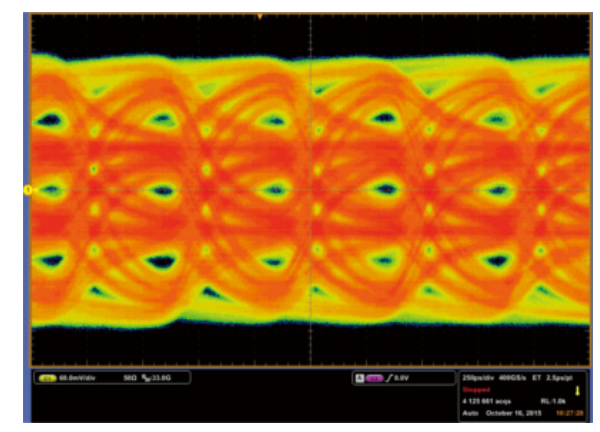

(a) Without THP

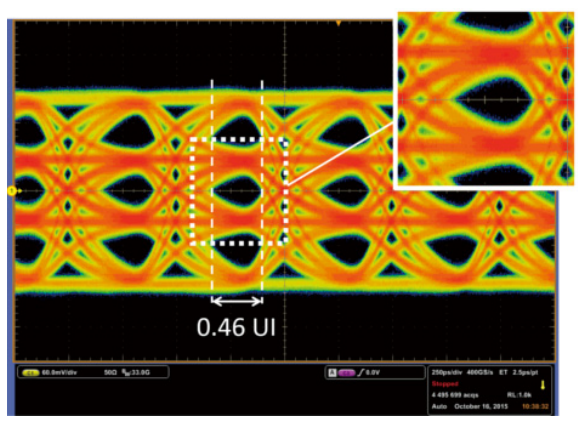

(b) With conventional THP

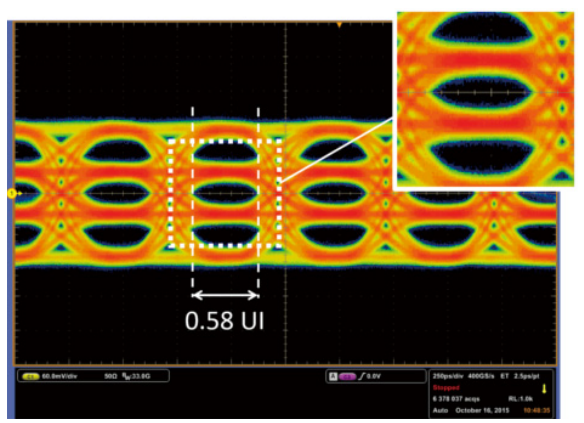

(c) With double-rate THP

Fig. 8 Measurement results of the eye diagram at 2 Gsps PAM-4 on a 1 m MSL.

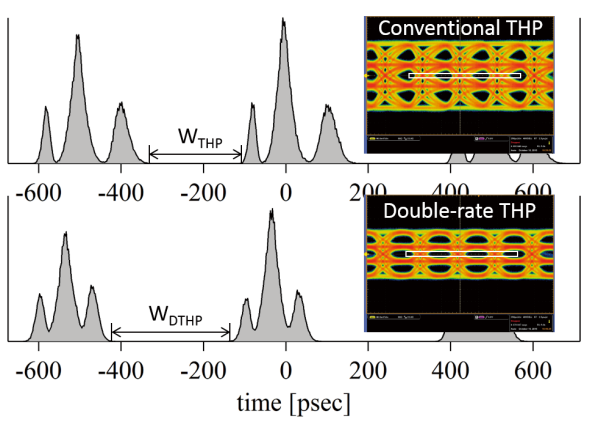

Fig. 9 Comparison of the timing margin. 
the transition time and, therefore, improve the eye width (Fig. 8(c)). The eye width of the double-rate THP is $0.58 \mathrm{UI}$, exhibiting a $26 \%$ improvement in the timing margin compared to conventional THP. Figure 9 shows the histogram of the eye width corresponding to the probability-density func-

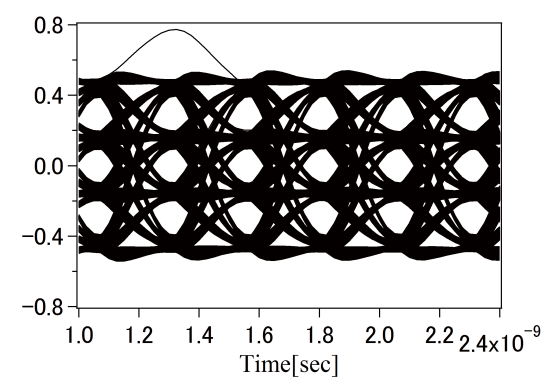

(a) Simulation result

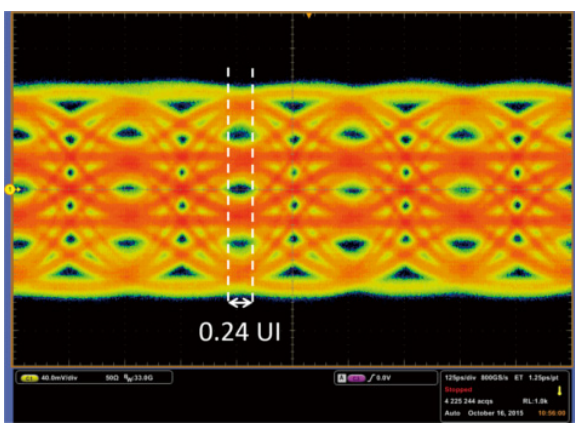

(b) Measurement results

Fig. 10 Eye diagram at 4 Gsps PAM-4 on a 1 m MSL using conventional THP.

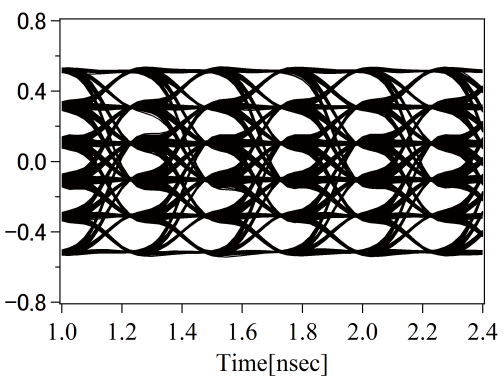

(a) Simulation result

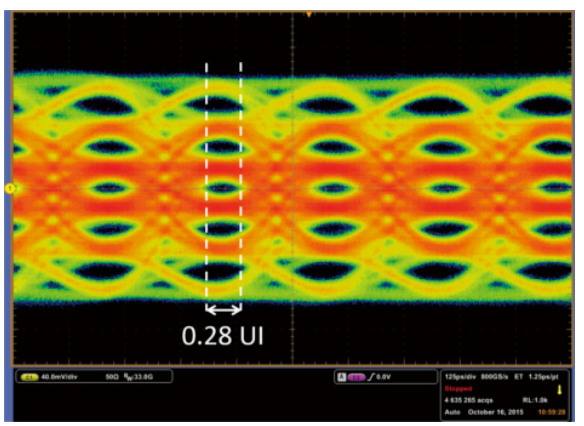

(b) Measurement results

Fig. 11 Eye diagram at 4 Gsps PAM- 4 on a $1 \mathrm{~m}$ MSL using double-rate THP. tion (PDF). As shown in Fig. 9, the transition times are reduced by applying the double-rate THP, which improves the eye width. The distribution of the PDF directly corresponds to the bit error rate (BER). Usually, an acceptable BER of $10^{-12}$ corresponding to a $7 \sigma$ distribution on the PDF, is used to estimate the signal integrity. The eye width can be expanded by using double-rate THP. The eye widths under the same PDF are $\mathrm{W}_{\mathrm{THP}}$ and $\mathrm{W}_{\mathrm{DTHP}}$, respectively, as shown in Fig. 9.

Figure 10 shows the simulation and measured results of eye diagrams of PAM-4 at 4 Gsps on a $1 \mathrm{~m} \mathrm{MSL} \mathrm{(a} 2$ times faster data rate than that shown in Fig. 8). The eye width of the conventional THP is 0.24 UI (Fig. 10(b)). Even though the received waveform using double-rate THP becomes multilevel because of the modulo operation, the eye width of Fig. 11(b) is improved to $0.28 \mathrm{UI}$.

\section{Discussion of Double-Rate THP}

As shown in previous simulation and measurement results, the proposed double-rate THP can greatly improve the eye compared to conventional THP. For example, the timing margin of the output of the double-rate THP is improved to $26 \%$ compared to conventional THP (Fig. 8). These experimental results show that double-rate THP is very effective in improving the timing margin of multi-valued data transmission by enhancing the transition edge.

Figure 12 shows a comparison between the conventional and double-rate THP effects. In the case of conventional THP, ISI is removed at the center symbol decision timing. Even though we can judge symbols precisely at decision timing, they are affected by ISI due to timing jitter. Conversely, double-rate THP can remove ISIs effectively on either side of decision timing at double the symbol rate. Therefore, double-rate THP can expand the eye, which contributes to the reduction of the ISI effect.

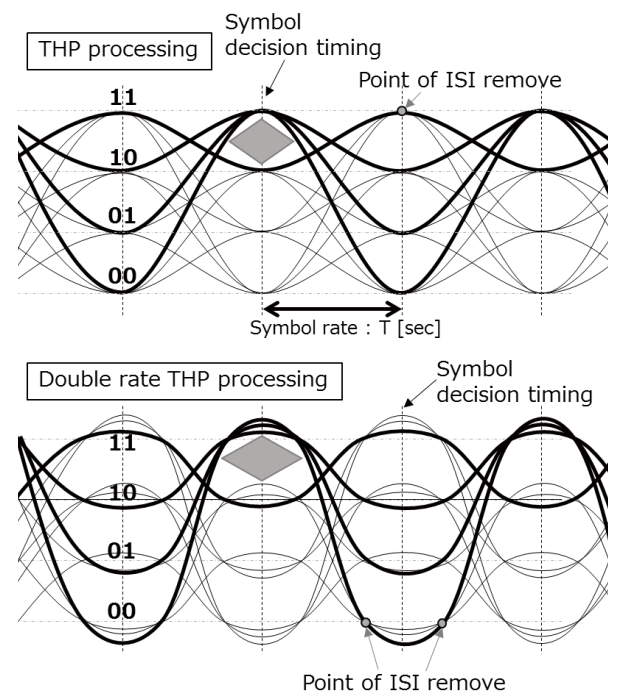

Fig. 12 Comparison between conventional and double-rate THP for PAM-4 signaling. 


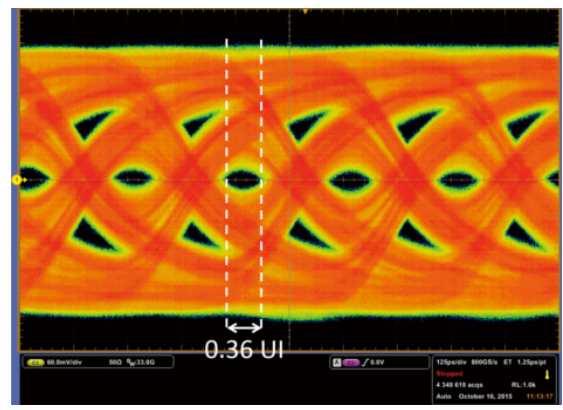

(a) Without THP

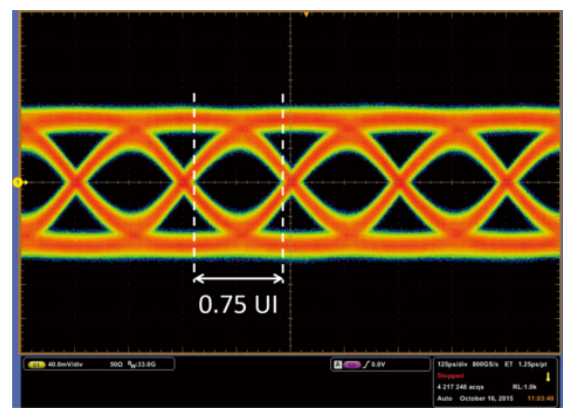

(b) With conventional THP

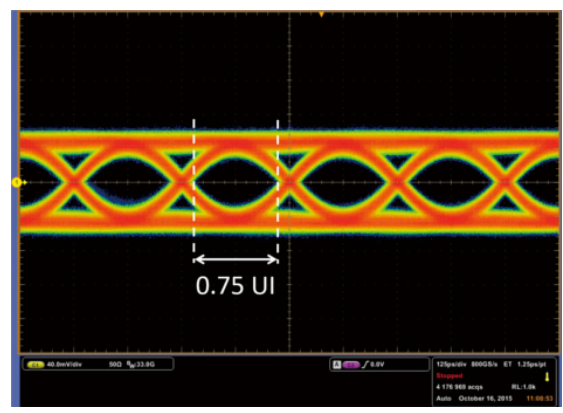

(c) With double-rate THP

Fig. 13 Measurement results of an eye diagram at 4 Gbps binary on a 2 m MSL.

Note that double-rate THP has a little effect on the binary data transmission. Figure 13 shows the measurement result of an eye diagram at 4 Gbps PAM-2 (binary) signaling data transmission using a $2 \mathrm{~m}$ MSL. The eye width is $0.36 \mathrm{UI}$ (Fig. 13(a)) without THP. The eye widths using the conventional THP and the double-rate THP are $0.75 \mathrm{UI}$ (Fig. 13(b)) and 0.75 UI (Fig. 13(c)), respectively. This is because the binary data transition pattern is simpler than PAM-4 and double sampling contributes little to the improvement of the eye width. Figure 14 explains the comparison between conventional and double-rate THP for binary data equalization. In the case of binary, cross points of data transition both $\mathbf{0}$ to $\mathbf{1}$ and $\mathbf{1}$ to $\mathbf{0}$ are nearly the same in both conventional and double-rate THP. This is because the binary data transmission combination is simple compared to the PAM-4 one.

In double-rate THP, due to the increase in the number of FIR filter taps, the adjustment of the THP coefficients becomes complex. Moreover, the channel characteristics

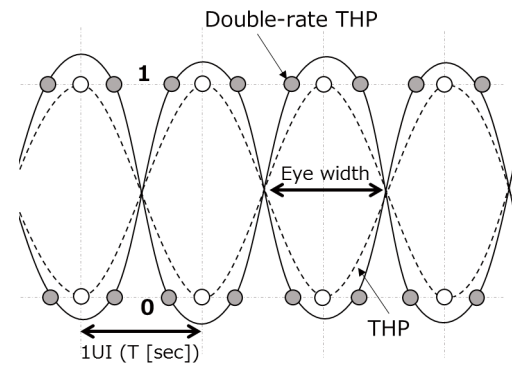

Fig. 14 Comparison between conventional and double-rate THP for binary signaling.

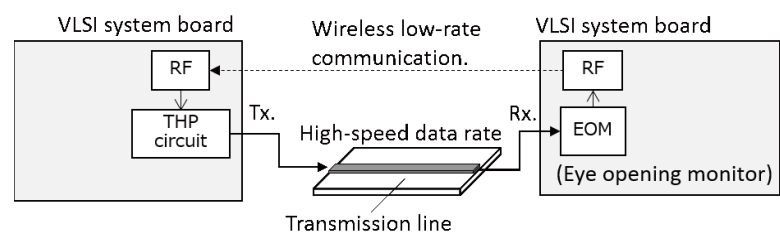

Fig. 15 Adaptive coefficient calibration.

Table 1 Number of taps of THP.

\begin{tabular}{|c||c|c|}
\hline Data rate, MSL length & THP & Double-rate THP \\
\hline 2 Gsps, 1-m MSL & 2 taps & 5 taps \\
\hline 4 Gsps, 1-m MSL & 5 taps & 11 taps \\
\hline
\end{tabular}

may change due to PVT (process, voltage and temperature) variations. To cope with this coefficient setting, an adaptive coefficient adjustment method is proposed. As shown in Fig. 15, the proposed system adjusts the coefficients using low-power and low-rate wireless communication to provide feedback of the receiver status using an eye monitoring system (EOM) to the transmitter [9]. Using simple wireless auxiliary communication, such as Bluetooth, adaptive coefficient calibration against channel characteristic variations can be achieved.

One drawback of double-rate THP is the increase in the hardware cost. Table 1 shows the number of taps required for the THP circuit at each data rate and the MSL length. The number of taps and the critical path of the filter for double-rate THP are increased, which affect the hardware cost and power consumption. In addition, the inverse filter of the double-rate THP needs to operate at twice the sampling frequency of conventional THP. Due to doublerate operation, the power consumption also increases. In the CMOS process, the power consumption increases in proportion to the operation frequency. To reduce the operation frequency by half, to the same frequency as conventional THP, pipeline and parallelization techniques are very effective [8]. Nevertheless, double-rate THP requires double-rate operation to DAC, resulting in increasing power consumption. Therefore, it is a major issue to reduce the power consumption of DAC. As stated above, the penalties of hardware implementation and signal integrity are related to the transactions in the double-rate THP.

In relation to hardware costs, THP equalization can- 
not provide the best solution with respect to high-speed data transmission for all applications. Instead, at the expense of those penalties, THP is particularly suited for the following conditions and applications: (1) High-speed data transmission over channels with severe bandwidth limitation and/or strong post-cursor reflections that are difficult to equalize using analog implementations. (2) It is possible to use a pipeline operation to increase the throughput of the data transmission. (3) Furthermore, because THP consists of digital rich ADC/DAC-based architecture, the THP performance can be improved by CMOS scaling. In addition, PVT variations can be adjusted using digital assistance.

\section{Conclusions}

This paper presented a new approach for high-speed data transmission using double-rate THP. First, the principle of the double-rate THP was explained and the effects of the equalization technique were simulated. Next, to evaluate the transmission characteristics, the improvement of PAM4 eye diagrams with double-rate THP was experimentally verified using MSLs.

Our experimental results show that the proposed double-rate THP is very effective for multi-valued data transmission. We demonstrated that the double-rate THP achieved a $26 \%$ improvement in the timing margin in PAM42 Gsps data transmission using a $1 \mathrm{~m}$ MSL. To show the superiority of the double-rate THP, comparisons with other transmitter equalization methods, such as FFE, are important. Future work will include comparisons under the same conditions.

\section{Acknowledgments}

This work was supported by JSPS KAKENHI Grant Numbers $15 \mathrm{H} 02675$ and $15 \mathrm{~K} 15962$.

\section{References}

[1] J.F. Bulzacchelli, "Equalization for Electrical Links," IEEE Solid State Circuits Mag., vol.7, no.4, pp.23-31, 2015.

[2] T. Kawamoto, T. Norimatsu, K. Kogo, F. Yuki, N. Nakajima, M. Tsuge, T. Usuji, T. Hokari, H. Koba, T. Komori, J. Nasu, T. Kawamata, Y. Ito, S. Umai, J. Kumazawa, H. Kurahashi, T. Muto, T. Yamashita, M. Hasegawa, and K. Higeta, "Multi-Standard $185 \mathrm{fs}_{r m s}$ 0.3-to-28Gb/s 40dB Backplane Signal Conditioner with Adaptive Pattern-Match 36-Tap DFE and Data-Rate-Adjustment PLL in 28nm CMOS," IEEE International Solid-State Circuit Conference, pp.54$55,2015$.

[3] Y. Iijima, Y. Takada, and Y. Yuminaka, "High-Speed Interconnection for VLSI Systems using Multiple-Valued Signaling with TomlinsonHarashima Precoding," IEICE Trans. on Information \& Systems, vol.E97-D, no.9, pp.2296-2303, 2014.

[4] Y.Iijima and Y.Yuminaka, "Evaluation of High-Speed Interfaces for VLSI Systems Using Tomlinson-Harashima Precoding," IEEE Proc. 44th International Symp. Multiple-Valued Logic, pp.138-143, May 2014.

[5] Y. Yuminaka and Y. Iijima, "Multiple-Valued Signaling for HighSpeed Serial Links Using Tomlinson-Harashima Precoding," IEEE J. Emerging and Selected Topics in Circuits and Systems, vol.6, no.1, pp.25-33, 2016

[6] M. Tomlinson, "New automatic equaliser employing modulo arithmetic," Electronics Letters, vol.7, pp.138-139, 1971.

[7] H. Harashima and H. Miyakawa, "Matched-transmission technique for channels with intersymbol interference," IEEE Trans. Commun., vol.COM-20, no.4, pp.774-780, 1972.

[8] M. Kossel, T. Toifl, P.A. Frances, M. Brandli, C. Menolfi, P. Buchmann, L. Kull, T.M. Andersen, and T. Morf, "A 10 Gb/s 8-Tap 6b 2-PAM/4-PAM Tomlinson-Harashima precoding transmitter for future memorylinkapplications in 22-nm SOI CMOS," IEEE J. solidstate circuits, vol.48, no.12, pp.3268-3284, 2013.

[9] B. Analui, A. Rylyakov, S. Rylov, M. Meghelli, and A. Hajimiri, "A $10-\mathrm{Gb} / \mathrm{s}$ two-dimensional eye-opening monitor in 0.13 -um standard CMOS," IEEE J. Solid-State Circuits, vol.40, no.12, pp.2689-2699, 2005.

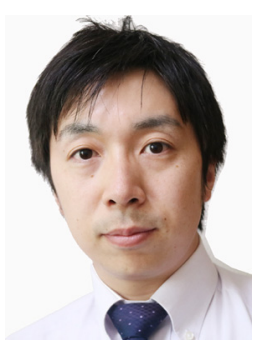

Yosuke Iijima received B. E. and M. E. degrees in electronic engineering from Gunma University, Kiryu, Japan, in 2003 and 2005, respectively, and received a D. E. degree in electronic engineering from the University of Tsukuba, Japan, in 2008. He is currently an Lecturer in the Department of Innovative Electrical and Electronic Engineering, National Institute of Technology, Oyama College, Oyama, Japan. His research interests include the design of high-speed interfaces, digital signal process-

ing and sensor communication systems and their applications.

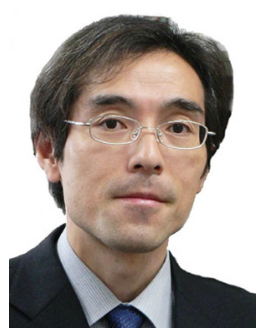

Yasushi Yuminaka received B. E., M. E., and D. E. degrees in electronic engineering from Tohoku University, Sendai, Japan, in 1990, 1992, and 1995, respectively. He is currently an Associate Professor in the Division of Electronics and Informatics, Graduate School of Science and Technology, Gunma University, Kiryu, Japan. His research interests include the design of multiple-valued integrated circuits, highspeed interfaces for VLSI systems, and newparadigm computing systems and their applications. Dr. Yuminaka received the IEE Ambrose Fleming Premium Award in 1994, the Niwa Memorial Award in 1995, the Young Engineer Award from the IEICE of Japan in 1995, the Excellent Poster Award from System LSI Biwako Workshop in 1999, the Young Excellent Author Award from Karuizawa Workshop in 2000 and the Outstanding Contributed Paper Award at the IEEE International Symposium on Multiple-Valued Logic in 2000 and 2009. He served as a Program Chair for IEEE International Symposium on Multiple-Valued Logic in 2013 and 2016. He is a member of IEEE and IEEJ. 